\title{
TABLE BASED ALGORITHM FOR INVERSE-TIME OVERCURRENT RELAY
}

\author{
Zoran N. Stojanović - Milenko B. Djurić
}

\begin{abstract}
This paper presents a new table based algorithm for overcurrent relay with inverse-time characteristic. The algorithm is based on loading the adequate time vector through which inverse-time characteristic is modeled. It uses samples of the current and calculates rms value. The rms current represents an input value for the index estimation what determines corresponding element from already loaded time vector. Since the calculations used in this algorithm are based on simple mathematical operations, short processing time is achieved. Performances of the algorithm are tested by several computer-generated signals. Furthermore, comparative analyses showed indisputably that suggested procedure possesses significant advantages compared to other solutions: simplicity, high speed of operation and accuracy.

K e y w o r d s: digital relaying, overcurrent protection, inverse-time characteristic, overcurrent relay
\end{abstract}

\section{INTRODUCTION}

Overcurrent protection represents one of the basic protections in every power system. Overcurrent and directional overcurrent relays are widely used for the protection of radial and ring subtransmission systems and distribution systems. They are also used as backup protection in transmission systems.

The input signal for this protection is the rms current of the protected element according to which tripping time of the relay is determined. There are two main mathematical methods for representing an overcurrent relay using digital computers: direct interpolation from stored data $[1,2]$ and use of data-fitting formulas. Direct data storage (table based algorithms) implies storing data in the memory of the computer for different time dial settings (TDS)/time multiplier settings (TMS) and then selecting operating points of a relay based on the stored data for different TDS/TMS. If the operating point does not match with one set of the stored values, then an interpolation is necessary to determine the corresponding time or TDS/TMS.

Regarding the data-fitting method, several equations have been suggested for representing the time-current characteristics of overcurrent relays [3-5]. These equations can be classified in two categories. The first category is that of the exponential equations and the second category is that of the polynomial equations.

In addition to conventional overcurrent relays which have fixed setting parameters, adaptive overcurrent relays [6-8] which can vary its setting parameters or its operating characteristics in response to changes in the power system are in use.

Recently, fuzzy logic and artificial neural networks are applied in overcurrent protection [9].

In this paper, IEEE standard C37.112-1996 [10] served as a base for development of the new algorithm. Accord- ing to this standard and its interpretation in [11], the overcurrent relay is described by tripping and reset characteristics with the following equations

$$
\begin{aligned}
t_{\text {trip }} & =\frac{A}{\left(I / I_{p u}\right)^{p}-1}+B, \\
t_{\text {res }} & =\frac{T_{\text {res }}}{\left(I / I_{p u}\right)^{2}-1},
\end{aligned}
$$

where $t_{\text {trip }}$ is tripping time and $t_{\text {res }}$ reset time in seconds, $I_{p u}$ is pick-up current, $I$ is input rms current $\left(I>I_{p u}-\right.$ tripping condition, $I \leq I_{p u}-$ reset condition), $A, B, p$ and $T_{\text {res }}$ are constants depending on the type of the characteristic and are shown in Table 1.

Table 1. Values of the standard constants A, B, p and $T_{\text {res }}$ regarding the type of the characteristic

\begin{tabular}{c|cccc}
\hline characteristic & $A$ & $B$ & $p$ & $T_{\text {res }}$ \\
moderately inverse & 0.0515 & 0.1140 & 0.02 & 4.85 \\
very inverse & 19.610 & 0.4910 & 2.00 & 21.6 \\
extremely inverse & 28.200 & 0.1217 & 2.00 & 29.1 \\
\hline
\end{tabular}

By discretization of mentioned characteristics for constant current increment, tripping/reset time vectors are obtained and memorized in the relay. The algorithm uses samples of the current to calculate rms value, which represents an input value for the index estimation from already loaded time vectors. Performances of the algorithm are tested by several computer-generated signals. In addition, comparative analyses showed indisputably that suggested procedure possesses significant advantages compared to other solutions: simplicity, high speed of operation and accuracy.

\footnotetext{
* Faculty of Electrical Engineering, University of Belgrade, Bulevar kralja Aleksandra 73, 11120 Belgrade, Serbia, stojanovic@etf.rs
} 


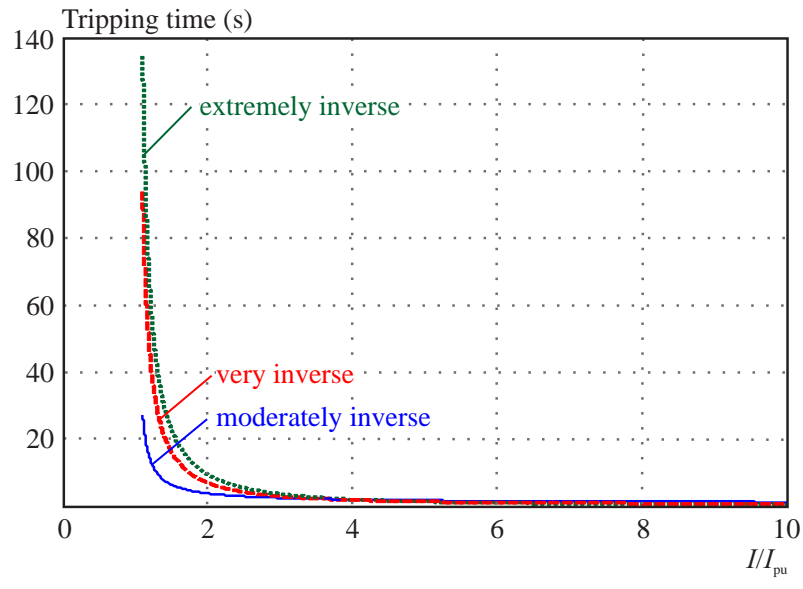

Fig. 1. The tripping characteristics

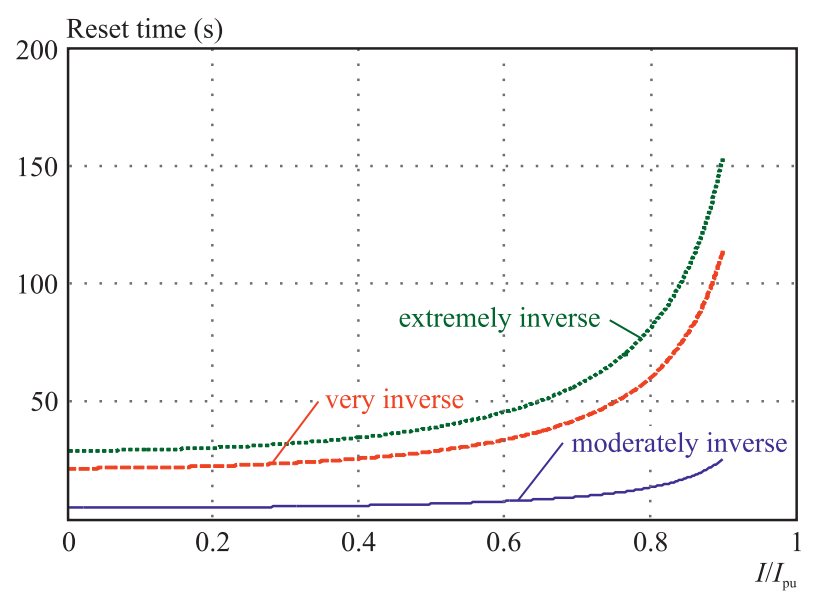

Fig. 2. The reset characteristics

Table 2. Discretization of moderately inverse tripping characteristic on the interval from $I_{\min }=1.1 I_{p u}$ to $I_{\max }=20 \mathrm{pu}: j-$ index of the vectors, $\boldsymbol{I}$ - vector of rms current in relative units, $\boldsymbol{t}_{\mathrm{trip}}$ - the tripping time vector in seconds

\begin{tabular}{c|cccccccc}
\hline$j$ & 1 & 2 & 3 & 4 & $\ldots$ & 482 & 483 & 484 \\
$I(j)$ & 1.100 & 1.139 & 1.178 & 1.217 & $\ldots$ & 19.907 & 19.947 & 19.986 \\
$t_{\text {trip }}(j)$ & 27.11 & 19.86 & 15.79 & 13.18 & $\ldots$ & 0.9494 & 0.9488 & 0.9483 \\
\hline
\end{tabular}

Table 3. Values of the vector $\boldsymbol{I}_{\text {modt }}$ for moderately inverse tripping characteristic on the interval from $I_{\min }=1.1 I_{p u}$ to $I_{\max }=20 \mathrm{pu}$ : $j$-index of the vectors, $\boldsymbol{I}_{\text {modt }}$ - the modified vector of the tripping current in relative units, $\boldsymbol{t}_{\text {trip }}$ - tripping time vector in seconds

\begin{tabular}{c|cccccccc}
\hline$j$ & 1 & 2 & 3 & 4 & $\ldots$ & 482 & 483 & 484 \\
$I_{\text {modt }}(j)$ & 1 & 2 & 3 & 4 & $\ldots$ & 482 & 483 & 484 \\
$t_{\text {trip }}(j)$ & 27.11 & 19.86 & 15.79 & 13.18 & $\ldots$ & 0.9494 & 0.9488 & 0.9483 \\
\hline
\end{tabular}

\section{FORMING OF THE TRIPPING AND RESET TIME VECTORS}

The tripping characteristics of the overcurrent relay for different values of constants $A, B$ and $p$ are given in Fig. 1.

The tripping time vector is formed by discretization of the characteristics presented in Fig. 1, using formula (1) for current increment $\Delta I$. As $\mathrm{A} / \mathrm{D}$ converter is a device with finite resolution, this fact is utilized for discretization of the characteristic in order to avoid the additional error. If this is taken into account, the current increment $\Delta I$ is

$$
\Delta I=\frac{2 I_{\max }}{2^{n_{c}}-1}
$$

where $I_{\max }$ is the maximal rms value of the input current and $n_{c}$ is the resolution of $\mathrm{A} / \mathrm{D}$ converter.

Number 2 in the numerator of (3) is the consequence of the alternating nature of the observed value, ie the current interval from $-I_{\max }$ to $I_{\max }$ is observed. $2^{n_{c}}-1$ in denominator represents the number of non-zero discrete levels or intervals which can be achieved with $n_{c}$ bits.

Let us assume that moderately inverse characteristic $(A=0.0515, B=0.1140, p=0.02), I_{p u}=1 \mathrm{pu}$ and 10 -bit $\mathrm{A} / \mathrm{D}$ converter are given. Discretization of this characteristic on the interval from $I_{\min }=1.1 I_{p u}$ to $I_{\max }=20 \mathrm{pu}$ will provide the results shown in Table 2 .

When the transformation (4) is applied on the vector I a new, modified vector is obtained

$$
\boldsymbol{I}_{\text {modt }}=\operatorname{round}\left(C\left(\boldsymbol{I}-I_{\min }\right)+1\right.
$$

where $\boldsymbol{I}_{\text {modt }}$ is the modified vector of the tripping current, $C$ is a constant which depends upon current increment $\Delta I$, that is $C=1 / \Delta I$ and round is the function of integer rounding.

Values of the modified vector of the tripping current are shown in Table 3.

If the obtained vectors $\boldsymbol{I}_{\text {modt }}$ and $\boldsymbol{t}_{\text {trip }}$ are inspected, the following could be observed: values of the vector $\boldsymbol{I}_{\text {modt }}$ are identical to indexes of the time vector $\boldsymbol{t}_{\text {trip }}$. That practically means that for every rms value of the input current $I$, which is higher or equal to $I_{\min }$, and lower or equal to $I_{\max }$, when formula (4) is applied, index of the vector $\boldsymbol{t}_{\text {trip }}$ could be determined. If the vector $\boldsymbol{t}_{\text {trip }}$ is formed in advance and memorized in the relay, it becomes unnecessary to calculate the complex formula (1) but only simple equation (4) which through indexes determines tripping time of the relay. In contrast to solutions presented in $[1,2]$ where it is necessary to conduct logarithmic calculations, here the same effect is achieved using simple mathematical operations such as integer rounding, multiplication and summation.

The same procedure could be applied for discretization of the reset characteristics of the relay which are depicted in Fig. 2.

In order to avoid negative reset time, equation (2) is corrected and the following is obtained

$$
t_{\mathrm{res}}=\frac{T_{\mathrm{res}}}{1-\left(I / I_{p u}\right)^{2}} \text {. }
$$

Discretization of the aforementioned characteristic during the interval from $I_{\operatorname{maxr}}=0.9 I_{p u}$ to 0 will provide the results shown in Table 4. 


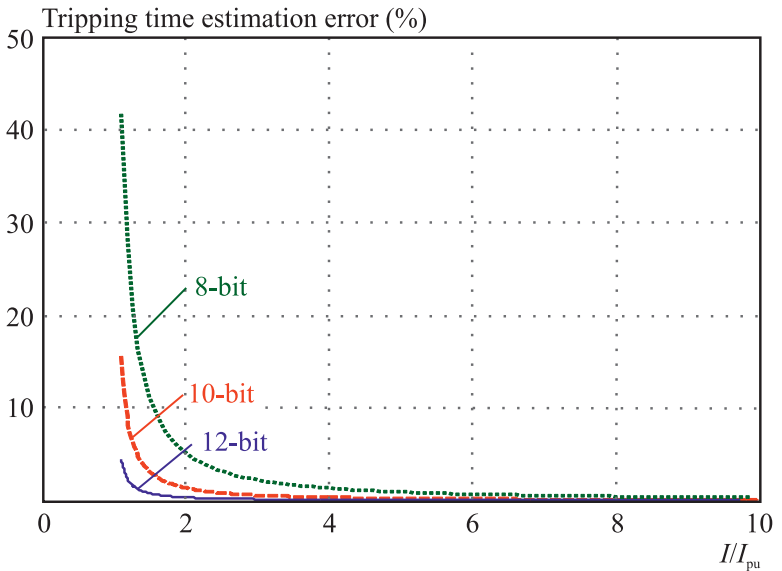

Fig. 3. The changes of the relative error of tripping time estimation in the function of rms current

Table 4. Discretization of moderately inverse reset characteristic during the interval from $I_{\operatorname{maxr}}=0.9 I_{p u}$ to $0: j-$ index of the vectors, $\boldsymbol{I}$ - the vector of rms current in relative units, $\boldsymbol{t}_{\mathrm{res}}-$ reset time vector in seconds

\begin{tabular}{c|cccccccc}
\hline$j$ & 1 & 2 & 3 & 4 & $\ldots$ & 22 & 23 & 24 \\
$I(j)$ & 0.900 & 0.861 & 0.822 & 0.783 & $\ldots$ & 0.079 & 0.040 & 0.001 \\
$t_{\mathrm{res}}(j)$ & 25.53 & 18.74 & 14.94 & 12.52 & $\ldots$ & 4.880 & 4.858 & 4.850 \\
\hline
\end{tabular}

Table 5. Values of the vector $\boldsymbol{I}_{\text {modr }}$ for moderately inverse reset characteristic during the interval from $I_{\operatorname{maxr}}=0.9 I_{p u}$ to $0: j-$ index of the vectors, $\boldsymbol{I}_{\text {modr }}$ - the modified vector of the reset current in relative units, $\boldsymbol{t}_{\mathrm{res}}$ - reset time vector in seconds

\begin{tabular}{c|cccccccc}
\hline$j$ & 1 & 2 & 3 & 4 & $\ldots$ & 22 & 23 & 24 \\
$I_{\operatorname{modr}}(j)$ & 1 & 2 & 3 & 4 & $\ldots$ & 22 & 23 & 24 \\
$t_{\mathrm{res}}(j)$ & 25.53 & 18.74 & 14.94 & 12.52 & $\ldots$ & 4.880 & 4.858 & 4.850 \\
\hline
\end{tabular}

Table 6. Discretization of very inverse tripping characteristic on the interval from $I_{\min }=1.1 I_{p u}$ to $I_{\max }=20 \mathrm{pu}: j-$ index of the vectors, $\boldsymbol{I}$ - the vector of rms current in relative units, $\boldsymbol{I}_{\text {modt }}$ - the modified vector of the tripping current in relative units, $\boldsymbol{t}_{\text {trip }}-$ tripping time vector in seconds

\begin{tabular}{c|cccccccc}
\hline$j$ & 1 & 2 & 3 & 4 & $\ldots$ & 482 & 483 & 484 \\
$I(j)$ & 1.100 & 1.139 & 1.178 & 1.217 & $\ldots$ & 19.907 & 19.947 & 19.986 \\
$I_{\text {modt }}(j)$ & 1 & 2 & 3 & 4 & $\ldots$ & 482 & 483 & 484 \\
$t_{\text {trip }}(j)$ & 93.87 & 66.40 & 51.01 & 41.19 & $\ldots$ & 0.5406 & 0.5404 & 0.5402 \\
\hline
\end{tabular}

Table 7. Discretization of very inverse reset characteristic during the interval from $I_{\operatorname{maxr}}=0.9 I_{p u}$ to $0: j-$ index of the vectors, $\boldsymbol{I}-$ the vector of rms current in relative units, $I_{\operatorname{modr}}-$ the modified vector of the reset current in relative units, $\boldsymbol{t}_{\text {res }}-$ reset time vector in seconds

\begin{tabular}{c|cccccccc}
\hline$j$ & 1 & 2 & 3 & 4 & $\ldots$ & 22 & 23 & 24 \\
$I(j)$ & 0.900 & 0.861 & 0.822 & 0.783 & $\ldots$ & 0.079 & 0.040 & 0.001 \\
$I_{\operatorname{modr}}(j)$ & 1 & 2 & 3 & 4 & $\ldots$ & 22 & 23 & 24 \\
$t_{\operatorname{res}}(j)$ & 113.68 & 83.45 & 66.53 & 55.76 & $\ldots$ & 21.735 & 21.634 & 21.600 \\
\hline
\end{tabular}

When the operation (6) is applied on the vector $\boldsymbol{I}$, the following is gained

$$
\boldsymbol{I}_{\operatorname{modr}}=\operatorname{round}\left(C\left(I_{\operatorname{maxr}}-\boldsymbol{I}\right)+1,\right.
$$

where $\boldsymbol{I}_{\text {modr }}$ is the modified vector of the reset current.

Values of the modified vector of the reset current are displayed in Table 5 .

As in the case of tripping characteristic, values of the vector $\boldsymbol{I}_{\mathrm{modr}}$ are identical to indexes of the time vector $\boldsymbol{t}_{\text {res }}$. Therefore, for every input value of rms current $I$, which is lower or equal to $I_{\operatorname{maxr}}$, index of the vector $\boldsymbol{t}_{\text {res }}$ could be determined when formula (6) is applied. If the vector $\boldsymbol{t}_{r e s}$ is formed in advance and memorized in the relay, it is not necessary to calculate complex formula (2) or (5), but only simple equation (6) which through indexes determines reset time of the relay. Compared to solutions given in $[1,2,12,13]$, where reset time is either 0 or constant, this algorithm varies reset time depending on the relay current.

This procedure is now applied to other two characteristics. Table 6 shows discretized very inverse tripping characteristic together with the modified current vector $\boldsymbol{I}_{\text {modt }}$ obtained by transformation (4). The corresponding discretized reset characteristic with the transformed current vector $\boldsymbol{I}_{\text {modr }}$ obtained by using formula (6) is shown in Table 7.

It can be observed from Tables 6 and 7 that transformations (4) and (6) are also applicable to very inverse characteristic since the modified current vectors $\boldsymbol{I}_{\text {modt }}$ and $\boldsymbol{I}_{\text {modr }}$ correspond to indexes $j$. This result has been expected since the formulas (4) and (6) are independent from the type of the characteristic.

Tables 8 and 9 relate to discretization of extremely inverse tripping and reset characteristics and contain values of the vector $\boldsymbol{t}_{\text {trip }}$ and $\boldsymbol{t}_{\text {res }}$ which are necessary to memorize when this characteristic is uploading.

Values from Tables 6-9 confirm earlier conclusions: for every rms value of the input current $I$, which is higher or equal to $I_{\min }$, and lower or equal to $I_{\max }$, when formula (4) is applied, index of the vector $\vec{t}_{\text {trip }}$ could be determined. On the other hand, for every input value of rms current $I$, which is lower or equal to $I_{\operatorname{maxr}}$, index of the vector $\boldsymbol{t}_{\text {res }}$ could be determined when formula (6) is applied. Additionally, it can be noted that the illustrated procedure is completely independent from the type of the characteristic and therefore applicable not only to exponential functions described by equations (1) and (2), but also to the characteristics of arbitrary complexity.

The reason for introducing current $I_{\text {min }}$ during tripping or $I_{\operatorname{maxr}}$ during reset lays in the inconclusiveness of tripping/reset time in the surrounding of $I_{p u}$. However, these intervals could be efficiently covered with constant tripping time for $I_{p u}<I<I_{\min }$, or constant reset time for $I_{\operatorname{maxr}}<I \leq I_{p u}$. The easiest way to accomplish this is to extend the integer rounding function round to nonnegative integer numbers.

Total dimension of both vectors $\boldsymbol{t}_{\text {trip }}$ and $\boldsymbol{t}_{\text {res }}$, depends only upon resolution of $\mathrm{A} / \mathrm{D}$ converter according to

$$
\operatorname{dim} \approx \frac{I_{\max }}{\Delta I}=\frac{2^{n_{c}-1}}{2},
$$




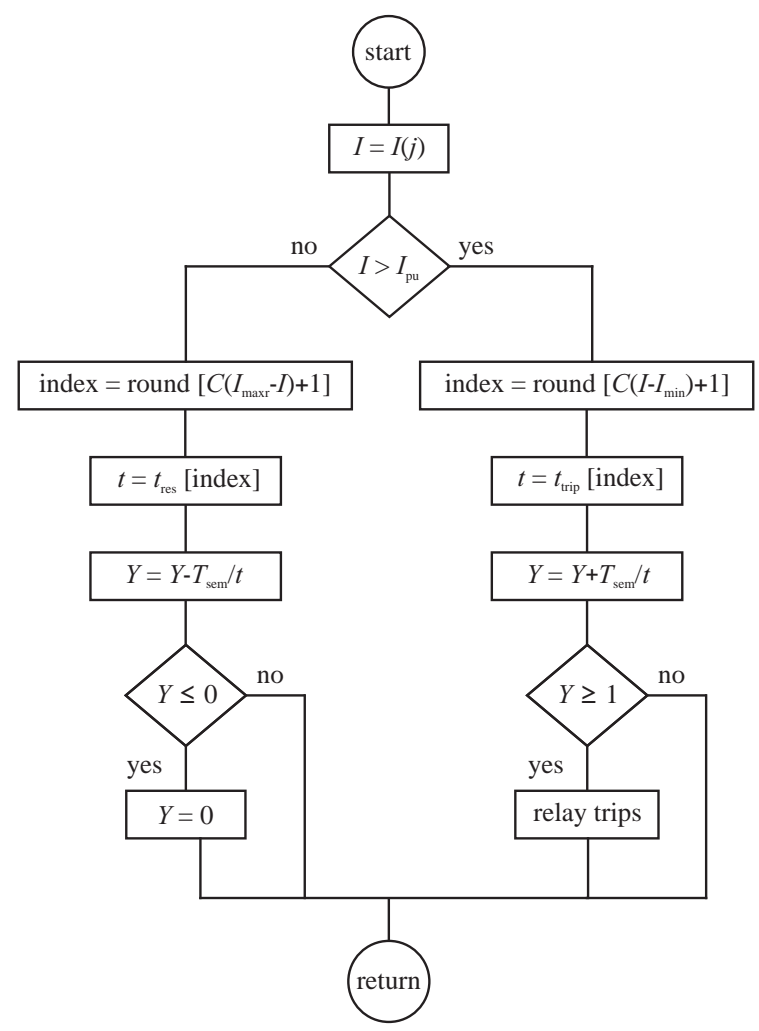

Fig. 4. The algorithm for the overcurrent relay

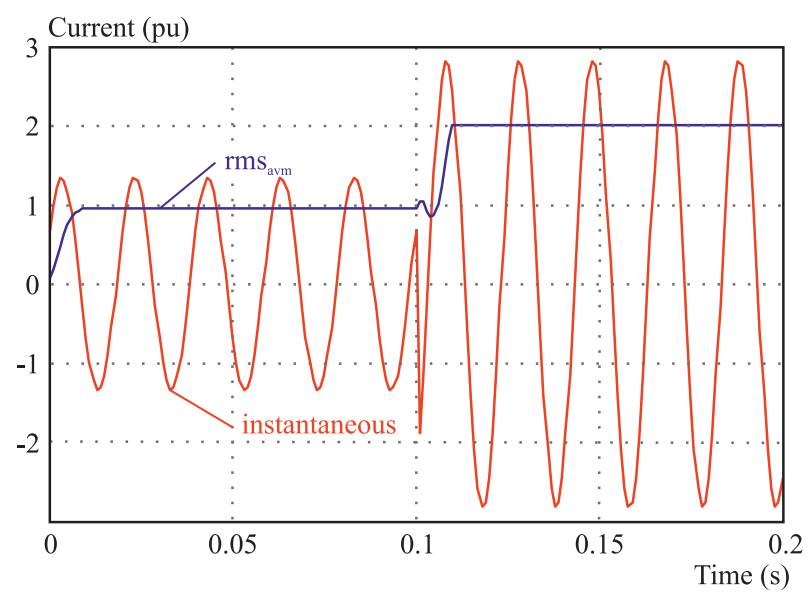

Fig. 5. The first test signal waveform and estimated rms current

while dimensions of each vector are also dependent on the pick-up current $I_{p u}$, but their sum remains constant for the same resolution of $\mathrm{A} / \mathrm{D}$ converter.

The error of tripping/reset time estimation is a consequence of the digitalization error of $\mathrm{A} / \mathrm{D}$ converter. This error depends on resolution of $\mathrm{A} / \mathrm{D}$ converter $n_{c}$ and on the interval which is discretized $\left(I_{\max }\right)$. When the converter resolution is higher, the error is decreasing. Conversely, when the discretized interval is larger, the error also increases. The time estimation error is highest in the surrounding of current $I_{p u}$, and in contrast to other solutions [1] where it is maintained constant and maximal throughout the whole interval of the current, here it expoftially decreases to 0 as the tripping current is raising
(Fig. 3). This novel solution minimizes the error, consequently enhancing the precision of the algorithm, while requirements for memory resources are increased.

Table 8. Discretization of extremely inverse tripping characteristic on the interval from $I_{\min }=1.1 I_{p u}$ to $I_{\max }=20 \mathrm{pu}: j-$ index of the vectors, $\boldsymbol{I}$ - the vector of rms current in relative units, $\boldsymbol{I}_{\operatorname{modt}}$ the modified vector of the tripping current in relative units, $\boldsymbol{t}_{\text {trip }}-$ tripping time vector in seconds

\begin{tabular}{c|cccccccc}
\hline$j$ & 1 & 2 & 3 & 4 & $\ldots$ & 482 & 483 & 484 \\
$I(j)$ & 1.100 & 1.139 & 1.178 & 1.217 & $\ldots$ & 19.907 & 19.947 & 19.986 \\
$I_{\text {modt }}(j)$ & 1 & 2 & 3 & 4 & $\ldots$ & 482 & 483 & 484 \\
$t_{\text {trip }}(j)$ & 134.41 & 94.90 & 72.77 & 58.65 & $\ldots$ & 0.1930 & 0.1928 & 0.1925 \\
\hline
\end{tabular}

Table 9. Discretization of extremely inverse reset characteristic during the interval from $I_{\operatorname{maxr}}=0.9 I_{p u}$ to $0: j-$ index of the vectors, $\boldsymbol{I}$ - the vector of rms current in relative units, $\boldsymbol{I}_{\operatorname{modr}}-$ the modified vector of the reset current in relative units, $\boldsymbol{t}_{\mathrm{res}}-$ reset time vector in seconds

\begin{tabular}{c|cccccccc}
\hline$j$ & 1 & 2 & 3 & 4 & $\ldots$ & 22 & 23 & 24 \\
$I(j)$ & 0.900 & 0.861 & 0.822 & 0.783 & $\ldots$ & 0.079 & 0.040 & 0.001 \\
$I_{\operatorname{modr}}(j)$ & 1 & 2 & 3 & 4 & $\ldots$ & 22 & 23 & 24 \\
$t_{\text {res }}(j)$ & 153.16 & 112.42 & 89.64 & 75.12 & $\ldots$ & 29.282 & 29.146 & 29.100 \\
\hline
\end{tabular}

\section{TESTING OF THE AGORITHM}

In order to determine the performances of the suggested algorithm, several tests were conducted with computer-generated current signals. The main program is using current samples with known sampling frequency to estimate rms value applying the method of average values $[14,15]$. When applying this method the basic assumption is that signals have sine or cosine waveforms. Then the average value of the current $I_{\mathrm{avg}}$ is

$$
\begin{array}{r}
I_{\mathrm{avg}}=\frac{1}{T} \int_{t}^{t+T}|i(t)| \mathrm{d} t=\frac{1}{T} \int_{t}^{t+T}\left|I_{m} \sin (\omega t+\varphi)\right| \mathrm{d} t \\
=\frac{2}{T} \int_{t}^{t+\frac{T}{2}}\left|I_{m} \sin (\omega t+\varphi)\right| \mathrm{d} t=\frac{2 I_{m}}{\pi},
\end{array}
$$

where $T$ is the basic period of the current signal, $I_{m}$ is magnitude of the current signal.

On the other hand, for rms value of the current $I$ stands

$$
I=\sqrt{\frac{1}{T} \int_{t}^{t+T} i(t)^{2} \mathrm{~d} t}=\sqrt{\frac{1}{T} \int_{t}^{t+T} I_{m}^{2} \sin ^{2}(\omega t+\varphi) \mathrm{d} t}=\frac{I_{m}}{\sqrt{2}}
$$

Combining equations (8) and (9) and switching from continuous to discrete system (the integral becomes the sum) the following is gained

$$
I(j)=\frac{\pi I_{\mathrm{avg}}(j)}{2 \sqrt{2}}=\frac{\pi}{m \sqrt{2}} \sum_{k=1}^{m / 2}|i(k)|
$$




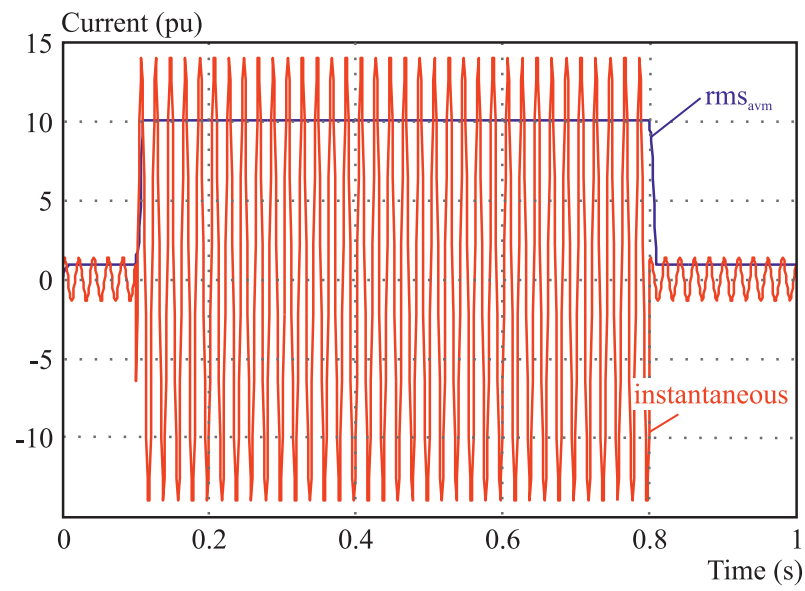

Fig. 6. The algorithm for the overcurrent relay

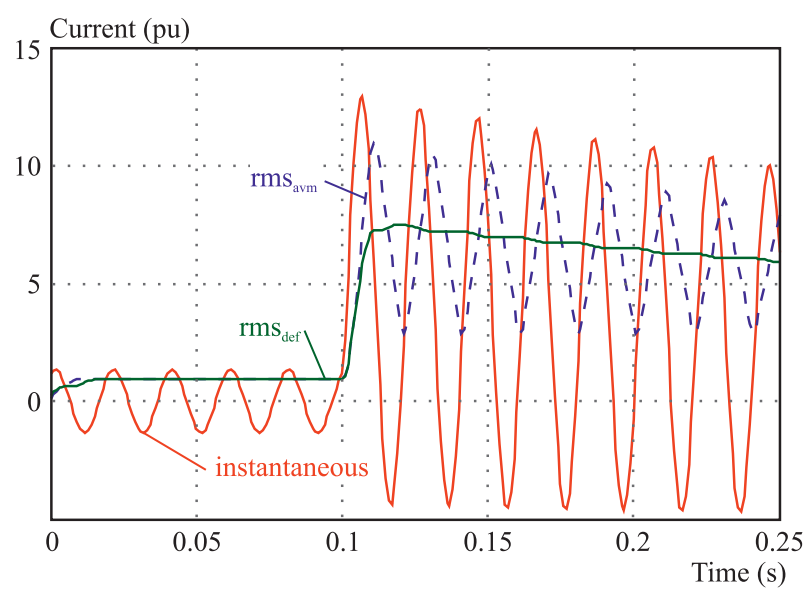

Fig. 7. The first test signal waveform and estimated rms current

where $m$ is the number of samples within the basic period of the signal and $i(k)$ are current samples.

Current samples are stored in the input register with the length of $m / 2$ samples (data window). After each sample is read-in, the left shift for one position is taking place in the register (the second sample becomes first, while the new sample becomes $m / 2$-th sample).

Applying the average value method, processing time for rms value calculation is minimized since the only operations in use are summation and multiplication. The estimation time of the rms value corresponds to one semiperiod $(10 \mathrm{~ms})$, ie to the time necessary for loading the input register with current samples.

When rms current higher then $I_{p u}$ appears, the program determines the index in every cycle and reads the adequate time from vector $\boldsymbol{t}_{\text {trip }}$. According to $[12,16]$, relay tripping condition is

$$
\sum_{1}^{N} \frac{T_{\text {sem }}}{t_{\text {trip }}}+Y \geq 1,
$$

where $T_{\text {sem }}$ is the sampling rate of $\mathrm{A} / \mathrm{D}$ converter and $Y$ is a cumulative counter.

Similarly to the previous, relay reset condition is

$$
Y-\sum_{1}^{N} \frac{T_{\text {sem }}}{t_{\text {res }}} \leq 0 .
$$

By introducing the cumulative counter $Y$, the system state before the fault is considered, ie the dynamic model of overcurrent relay is obtained.

Table 10. Results of the first test: $I_{\text {trip }}$ - estimated current when relay trips, $t$ - tripping time, $t^{\prime}$ - tripping time according to (1) for real value of the fault current, $G_{t}$ - relative error of the tripping time estimation

\begin{tabular}{c|cccc}
\hline characteristic & $I_{\text {trip }}$ & $t$ & $t^{\prime}$ & $G_{t}$ \\
& $(\mathrm{pu})$ & $(\mathrm{s})$ & $(\mathrm{s})$ & $(\%)$ \\
moderately inverse & 2.005 & 3.785 & 3.803 & 0.47 \\
very inverse & 2.005 & 6.960 & 7.028 & 0.97 \\
extremely inverse & 2.005 & 9.422 & 9.522 & 1.05 \\
\hline
\end{tabular}

Table 11. Results of the second test: $I_{\text {trip }}$ - estimated current when relay trips, $t$ - tripping time, $t^{\prime}$ - tripping time according to (1) for real value of the fault current, $G_{t}$ - relative error of the tripping time estimation

\begin{tabular}{c|cccc}
\hline characteristic & $I_{\text {trip }}$ & $t$ & $t^{\prime}$ & $G_{t}$ \\
& $(\mathrm{pu})$ & $(\mathrm{s})$ & $(\mathrm{s})$ & $(\%)$ \\
moderately inverse & $/$ & $/$ & 1.208 & $/$ \\
very inverse & 10.041 & 0.694 & 0.689 & 0.73 \\
extremely inverse & 10.041 & 0.411 & 0.407 & 0.98 \\
\hline
\end{tabular}

For values of the input current between $I_{p u}$ and $I_{\min }$ the relay trips with constant time delay which corresponds to the current $I_{\min }$. As in the case of tripping, for the currents between $I_{\operatorname{maxr}}$ and $I_{p u}$ relay resets after constant time which corresponds to the current $I_{\operatorname{maxr}}$, while for the currents lower then $I_{\text {maxr }}=0.9 I_{p u}$ gradual reset of the relay occurs by reading the adequate times from the vector $\boldsymbol{t}_{\text {res }}$. Described algorithm is depicted in Fig. 4.

Figure 5 shows the input current signal which was used for the first test and estimated rms current obtained by the method of average values ( $\left.\mathrm{rms}_{\mathrm{avm}}\right)$. Adjusted rms current before the fault is $0.95 \mathrm{pu}$, and after the fault $2 \mathrm{pu}$. Adopted value of the inception angle is $\theta=\pi / 6 \mathrm{rad}$ and the fault instant is $0.1 \mathrm{~s}$ after the beginning of the simulation. For the sampling frequency the value of $f_{\mathrm{sem}}=1 \mathrm{kHz}\left(T_{\mathrm{sem}}=1 / f_{\mathrm{sem}}=1 \mathrm{~ms}\right)$ was taken [17]. In addition, the following adjustments of the relay were applied: $n_{c}=12, I_{p u}=1 \mathrm{pu}, I_{\max }=20 \mathrm{pu}$.

Estimated rms current at which the relay trips $I_{\text {trip }}$ and relay tripping time $t$ for different tripping characteristics are given in Table 10.

It can be concluded from Table 10 that estimated current obtained by the average values method $I_{\text {trip }}$ is the same in all three cases (2.005 pu) and represents only negligible variation compared to the real value which is $2 \mathrm{pu}$. In order to consider the error which relay creates while estimating tripping time, the exact value of tripping time $t^{\prime}$ is calculated according to the equation (1) for the real 


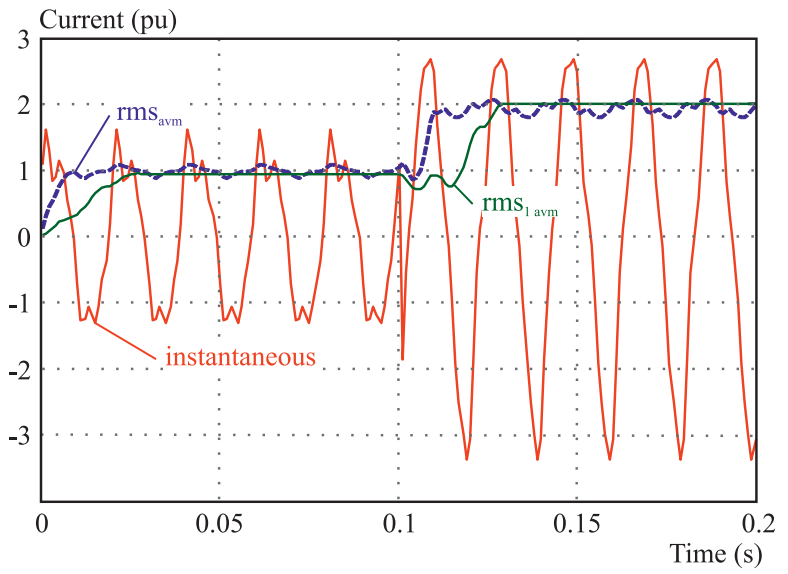

Fig. 8. The fourth test signal waveform and estimated rms currents

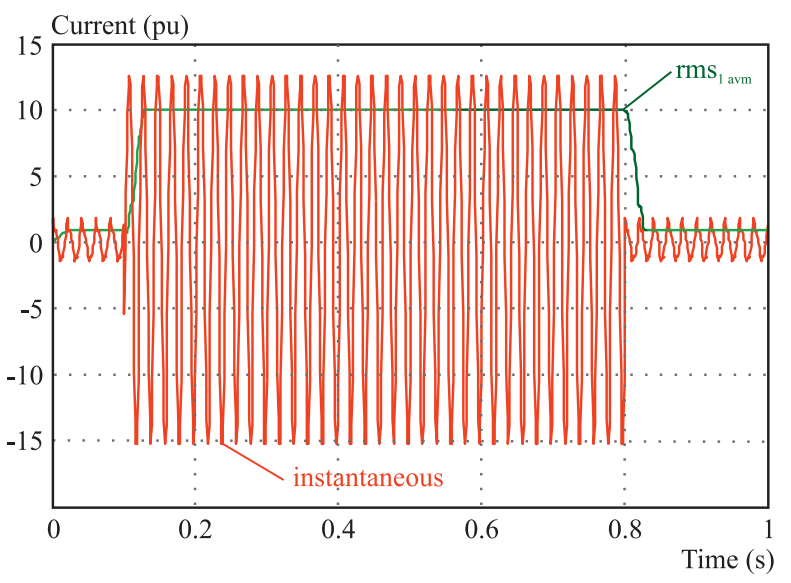

Fig. 9. The fifth test signal waveform and estimated rms currents

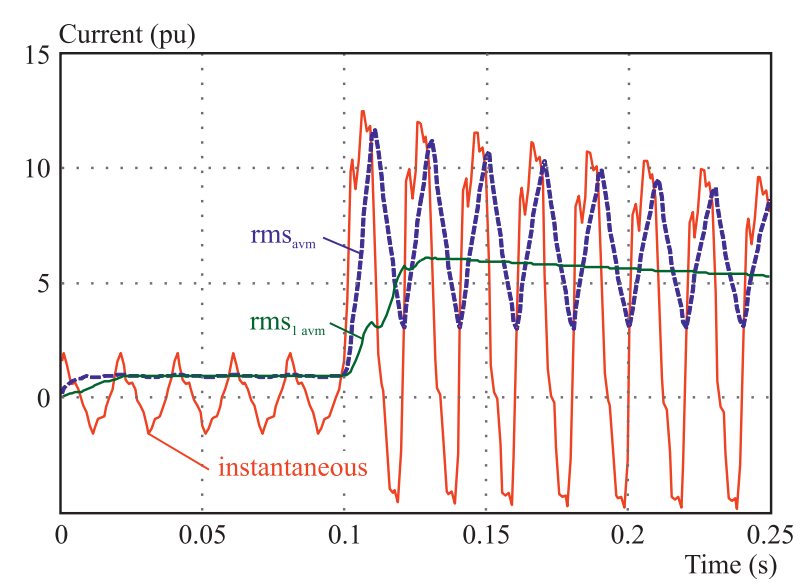

Fig. 10. The sixth test signal waveform and estimated rms currents

current value and given in Table 10. As the relative error of the time estimation $G_{t}$ is around $1 \%$, it can be stated that the suggested technique possesses quite satisfactory precision.

For the second test, the input current signal and estimated rms value are shown in Fig. 6. Adjusted rms value of the current during the fault is $10 \mathrm{pu}$, while the fault lasts for $0.7 \mathrm{~s}$. Adopted value of the inception angle is $\theta=\pi / 4 \mathrm{rad}$.
The results of the second test for different tripping characteristics are given in Table 11. The relay does not trip when moderately inverse characteristic is used because the duration of the fault is shorter then the projected tripping time (around $1.2 \mathrm{~s}$ ). In other cases relay trips and the relative error of the tripping time estimation is below $1 \%$.

In the third test, the magnitude of fault current is changing over time (Fig. 7). Adopted value of the inception angle is $\theta=\pi / 3 \mathrm{rad}$. As the fault current also contains decreasing direct component, application of the average values method for estimating rms current results in a significant error (estimated current according to this method is marked as $\mathrm{rms}_{\text {avm }}$ on Fig. 7). Significant error is the reason why in this test equation (9) is used for calculating rms current. Transformation of this equation from continuous to discrete domain gives

$$
I_{\mathrm{def}}(j)=\sqrt{\frac{1}{T} \sum_{k=1}^{m} i(k)^{2} T_{\mathrm{sem}}}=\sqrt{\frac{1}{m} \sum_{k=1}^{m} i(k)^{2}} .
$$

Estimated rms current according to (13) on Fig. 7 is marked as $\mathrm{rms}_{\mathrm{def}}$. The results of the third test for various tripping characteristics are given in Table 12. In this test calculation of the relative error is pointless, because the exact tripping time $t^{\prime}$ is possible to determine only for constant rms current.

Since raw signals might contain high-order harmonics and/or direct component, it is convenient to filter samples before usage. If the cosine Fourier series is used [14,15], the $m$-th filtered current sample is gained through equation

$$
i_{f}(m)=\frac{2}{m} \sum_{k=1}^{m} i(k) \cos \frac{2 \pi k}{m}
$$

Since filtering introduces additional delay which corresponds to the basic period of the signal, total convergence time of the rms value is now $30 \mathrm{~ms}$ (Fourier filter requires $20 \mathrm{~ms}$, the average values method $10 \mathrm{~ms}$ ).

Now, previous three tests are repeated but with adding high-order harmonics in the input current. The following high-order harmonics are adopted: $I_{2}=10 \% I_{1}, I_{3}=$ $20 \% I_{1}, I_{4}=5 \% I_{1}$, and $I_{5}=15 \% I_{1}$, with the inception angles: $\theta_{2}=\pi / 2 \mathrm{rad}, \theta_{3}=\pi / 3 \mathrm{rad}, \theta_{4}=\pi / 4 \mathrm{rad}$, and $\theta_{5}=0 \mathrm{rad}$

Figures 8, 9 and 10 show the input current signals and estimated rms currents. For easier comparison, estimated rms values obtained before ( $\left.\mathrm{rms}_{\mathrm{avm}}\right)$ and after filtering $\left(\mathrm{rms}_{1 \mathrm{avm}}\right)$ are displayed at the same Figure. The results of the tests for different tripping characteristics are given in Tables 13, 14 and 15 . 
Table 12. Results of the third test: $I_{\text {trip }}$ - estimated current when relay trips, $t$ - tripping time

\begin{tabular}{c|cc}
\hline characteristic & $I_{\text {trip }}$ & $t$ \\
$(\mathrm{pu})$ & $(\mathrm{s})$ \\
moderately inverse & 2.095 & 2.522 \\
very inverse & 2.046 & 3.143 \\
extremely inverse & 2.017 & 4.044 \\
\hline
\end{tabular}

Table 13. Results of the fourth test: $I_{\text {trip }}$ - estimated current when relay trips, $t$ - tripping time, $t^{\prime}$ - tripping time according to (1) for real value of the fault current, $G_{t}$ - relative error of the tripping time estimation

\begin{tabular}{c|cccc}
\hline characteristic & $I_{\text {trip }}$ & $t$ & $t^{\prime}$ & $G_{t}$ \\
& $(\mathrm{pu})$ & $(\mathrm{s})$ & $(\mathrm{s})$ & $(\%)$ \\
moderately inverse & 2.005 & 3.785 & 3.803 & 0.47 \\
very inverse & 2.005 & 6.960 & 7.028 & 0.97 \\
extremely inverse & 2.005 & 9.423 & 9.522 & 1.04 \\
\hline
\end{tabular}

By applying the Fourier filter the error of tripping time estimation slightly increases, since the additional delay of $20 \mathrm{~ms}$ is introduced. However, the algorithm still possesses satisfactory accuracy. Also, the procedure is now applicable even in conditions of distorted input signals.

In order to realize advantages of suggested algorithm compared to known solutions, processing time for each algorithm is tested. The following algorithms are considered:

1. table based algorithm presented in this paper,

2. log-table based algorithm presented in $[1,2]$,

3. data-fitting method using exponential equation (1),

4. data-fitting method using polynomial equation

$$
t_{\text {trip }}=a_{0}+\frac{a_{1}}{I-1}+\frac{a_{2}}{(I-1)^{2}}+\frac{a_{3}}{(I-1)^{3}}+\frac{a_{4}}{(I-1)^{4}} \text {, }
$$

5. data-fitting method using log-polynomial equation

$$
t_{\text {trip }}=a_{0}+\frac{a_{1}}{\log I}+\frac{a_{2}}{(\log I)^{2}}+\frac{a_{3}}{(\log I)^{3}}+\frac{a_{4}}{(\log I)^{4}} \text {. }
$$

Compared to original equations given in [9] and $[6]$ polynomial equations (15) and (16) are simplified by neglecting terms with an exponent greater than 4 .

Comparison tests are performed under the same conditions on the personal computer with the following characteristics: AMD Athlon 64 X2 Dual Core Processor 5200+, 2.71 GHz, 64-bit, 1.75 GB of RAM. Each method is executed 1000000 times and calculation time is measured. Along with obtained absolute units, the results are also presented in relative units compared to the execution time of the new algorithm (Table 16).

If the table based algorithms are compared, it becomes obvious that the algorithm presented in this paper requires less processing time. In addition, a smaller error in time estimation places this algorithm ahead of log-table based algorithm. Furthermore, it should be noted that log-table algorithm can not support reset characteristic and therefore dynamic performance of the relay is lost. The only drawback of the new approach is enhanced demand for memory resources compared to log-table based algorithm.

Compared to data-fitting approaches, new algorithm possesses higher speed of performance. This improvement becomes even more dominant as the formula for datafitting becomes more complex. Another advantage compared to the extrapolation techniques is that the processing time of new algorithm is independent of the characteristic complexity. Furthermore, new technique is not introducing error while estimating time. The only error is a consequence of the $\mathrm{A} / \mathrm{D}$ converter digitalization. On the other hand, it is necessary to reach a compromise when using data-fitting methods: simpler formula requires less usage of CPU time, while the precision in curve fitting is decreasing. Conversely, more complex formula occupies more of the CPU time but the tripping/reset time estimation is more accurate.

Table 14. Results of the fifth test: $I_{\text {trip }}$ - estimated current when relay trips, $t$ - tripping time, $t^{\prime}$ - tripping time according to (1) for real value of the fault current, $G_{t}$ - relative error of the tripping time estimation

\begin{tabular}{c|cccc}
\hline characteristic & $I_{\text {trip }}$ & $t$ & $t^{\prime}$ & $G_{t}$ \\
& $(\mathrm{pu})$ & $(\mathrm{s})$ & $(\mathrm{s})$ & $(\%)$ \\
moderately inverse & $/$ & $/$ & 1.208 & $/$ \\
very inverse & 9.879 & 0.699 & 0.689 & 1.45 \\
extremely inverse & 10.041 & 0.418 & 0.407 & 2.70 \\
\hline
\end{tabular}

Table 15. Results of the sixth test: $I_{\text {trip }}$ - estimated current when relay trips, $t$ - tripping time

\begin{tabular}{c|cc}
\hline characteristic & $I_{\text {trip }}$ & $t$ \\
$(\mathrm{pu})$ & $(\mathrm{s})$ \\
moderately inverse & 2.094 & 2.581 \\
very inverse & 2.038 & 3.464 \\
extremely inverse & 2.013 & 4.795 \\
\hline
\end{tabular}

\section{CONCLUSION}

Displayed algorithm represents novel solution for the relay operation with inverse-time characteristic on table based principle. The method is based on loading the adequate time vector through which inverse-time characteristic is modeled. It uses the current samples to calculate rms value which represents an input for the index estimation what determines corresponding element from already loaded time vector. For designing of the given algorithm simple mathematical operations are used such as integer rounding, multiplication and summation. Due to its simplicity, the high speed of operation is achieved. The error of the tripping/reset time estimation is adapted to the digitalization error of the A/D converter. Conducted side-by-side analysis highlighted all the advantages of suggested procedure compared to the other solutions: simplicity, short processing time and accuracy. Furthermore, 
Table 16. Processing time for different types of algorithm

\begin{tabular}{c|ccccc}
\hline algorithm & $\begin{array}{c}\text { new table } \\
\text { based }\end{array}$ & $\begin{array}{c}\text { log-table } \\
\text { based }\end{array}$ & $\begin{array}{c}\text { data-fitting } \\
\text { using (1) }\end{array}$ & $\begin{array}{c}\text { data-fitting } \\
\text { using (15) }\end{array}$ & $\begin{array}{c}\text { data-fitting } \\
\text { using (16) }\end{array}$ \\
processing time (s) & 3.25 & 7.78 & 4.13 & 6.84 & 10.78 \\
processing time (\%) & 100.00 & 239.42 & 126.92 & 210.58 & 331.72 \\
\hline
\end{tabular}

described method for forming the tripping/reset time vectors is applicable not only to already mentioned characteristics but to characteristics of arbitrary complexity without any additional demands.

\section{REFERENCES}

[1] BENMOUYAL, G.: A Log-Table Based Algorithm for Implementing Microprocessor Time-Overcurrent Relays, IEEE Transactions on Power Apparatus and Systems 101 (Sep 1982), 3563-3567.

[2] Benmouyal, G.: Design of a Digital Multi-Curve TimeOvercurrent Relay, IEEE Transactions on Power Delivery 5 (Oct 1990), 1725-1731.

[3] IEEE Committee Report: Computer Representation of Overcurrent Relay Characteristics, IEEE Transactions on Power Delivery 4 (July 1989), 1659-1667.

[4] CHERYL, A. K.-WALTER, A. E. : Flexible Inverse Overcurrent Relaying using a Microprocessor, IEEE Transactions on Power Delivery 5 (Apr 1990), 915-923.

[5] MURTY, V.-YALLA, V. S. : A Digital Multifunction Protective Relay, IEEE Transactions on Power Delivery 7 (Jan 1992), 193-201.

[6] ENRIQUeZ, A. C.-MARTINEZ, E. V.-FERRER, H. J. A.: Time Overcurrent Adaptive Relay, Electrical Power and Energy Systems 25 (Dec 2003), 841-847.

[7] ENRIQUEZ, A. C.-MARTINEZ, E. V.: Sensitivity Improvement of Time Overcurrent Relay, Electric Power Systems Research $\mathbf{7 7}$ (Feb 2007), 119-124.

[8] YONGLI, Z.-SHAOQUN, S.-DEWEN, W.: MultiagentsBased Wide Area Protection with Best-Effort Adaptive Strategy, Electrical Power and Energy Systems 31 (Feb 2009), 94-99.

[9] KAREGAR, H. K.-ABYANEH, H. A.-Al-DABBAGH, M. : A Flexible Approach for Overcurrent Relay Characteristics Simulation, Electric Power Systems Research 66 ( Sep 2003), 233-239.

[10] IEEE St. C37.112-1996 : IEEE Standard Inverse-Time Characteristic Equations for Overcurrent Relays, 1996.

[11] BENMOUYAL, G.-MEISINGER, M.-BURNWORTH, J.ELMORE, W. A.-FREIRICH, K.-KOTOS, P. A.-LEBLANC, P. R.-LERLEY, P. J.-McCONNELL, J. E.-MIZENER, J.-PINTO de sa-J.-RAMASWAMI, R.-SACHDEV, M. S.-STRANG, W. M.-WALDRON, J. E.-WATANSIRIROCH, S.-ZOCHOLL, S. E.: IEEE Standard Inverse-Time Characteristic Equations for Overcurrent Relays, IEEE Transactions on Power Delivery 14 (July 1999), 868-872.
[12] BENMOUYAL, G.: Some Aspects of the Digital Implementation of Protection Time Function, IEEE Transactions on Power Delivery 5 (Oct 1990), 1705-1713.

[13] GAN, Z.-ELANGOVAN, S.-LIEW, A. C. : Microcontroller Based Overcurrent Relay and Directional Overcurrent Relay with Ground Fault Protection, Electric Power Systems Research 38 (July 1996), 11-17.

[14] STOJANOviĆ, Z.-DJURIĆ, M.: The algorithm for Directional Element without Dead Tripping Zone Based on Digital Phase Comparator, Electric Power Systems Research 81 (Feb 2011), 377-383.

[15] STOJAnOvić, Z.-DJURIĆ, M.: An Algorithm for Directional Earth-Fault Relay with no Voltage Inputs, Electric Power Systems Research 96 (Mar 2013), 144-149.

[16] ZOCHOLL, S.-BENMOUYAL, G.: On the Protection of Thermal Processes, IEEE Transactions on Power Delivery 20 (Apr 2005), 1240-1246.

[17] McLAREN, P. G.-MUSTAPHI, K.-BENMOUYAL, G.CHANO, S.-GIRGIS, A.-HENVILLE, C.-KEZUNOVIC, M.-KOJOVIC, L.-MARTTILA, R.-MEISINGER, M.-MICHEL, G.-SACHDEV, M. S.-SKENDZIC, V.-SIDHU, T. S.-TZIOUVARAS, D.: Software Models for Relays, IEEE Transactions on Power Delivery 16 (Apr 2001), 238-245.

Received 25 March 2013

Zoran N. Stojanović born in 1979 in Požarevac, received BS, MSc and PhD from the Faculty of Electrical Engineering, University of Belgrade, Serbia in 2003, 2009 and 2012, respectively. From 2003 to 2004 he worked in the Department for Design at Energoprojekt-Hidroinženjering, Belgrade. Since 2005, he has been with the Faculty of Electrical Engineering, University of Belgrade and holds the position of Assistant Professor. His main fields of scientific interest are relay protection and monitoring of high voltage equipment.

Milenko B. Djurić born in 1948 in Sarajevo, received the $\mathrm{BS}, \mathrm{MSc}$ and $\mathrm{PhD}$ degrees in Electrical Engineering from the Faculty of Electrical Engineering (University of Belgrade/Serbia) in 1973, 1977 and 1985, respectively. A few years he spent in the Electric Power Company in Kraljevo/Serbia. In 1980 he jointed the Faculty of Electrical Engineering, Belgrade, where he is a full Professor. His main areas of scientific interests are power system protection and stability and digital signal processing applications in power system protection. 